\title{
Renal Interstitial Fibrosis Is Reduced in High- Fat Diet-Induced Obese Pigs following Renal Denervation from the Intima and Adventitia of the Renal Artery
}

\author{
Binbin Zhu ${ }^{a, b}$ Yahui Liu ${ }^{a, b}$ Datun Qi ${ }^{b}$ Linwei Zhao ${ }^{b}$ Xiaohang Yang ${ }^{b}$ \\ Enyong Su ${ }^{b}$ Zhongyu Zhu $^{b}$ You Zhang ${ }^{b}$ Chuanyu Gao ${ }^{a}$, b \\ aDepartment of Cardiology, Zhengzhou University People's Hospital, Henan Provincial People's Hospital, \\ Zhengzhou, China; ' Department of Cardiology, Henan Provincial Key Lab for Control of Coronary Heart Disease, \\ Central China Fuwai Hospital, Zhengzhou, China
}

\section{Keywords}

Renal denervation - Adventitia of the renal artery •

Hypertension · Glomerular injury · Fibrosis

\begin{abstract}
Background: This study aims to compare whether 2 different routes of renal denervation (RDN) from the intima and adventitia of the renal artery can reduce renal fibrosis in a pig model of hypertension induced by a high-fat diet and to explore possible molecular mechanisms. Methods: Twentyfour Bama miniature pigs were randomly divided into a control group (normal diet, $n=6$ ) or a hypertension model group (high-fat diet, $n=18$ ). The model group was randomly divided into the intima-RDN group $(n=6)$, the adventitia-RDN group $(n=6)$, or the renal arteriography-only group (sham group, $n=6$ ). All animals were fed separately. The model group was fed a high-fat diet after the operation, and the control group was fed conventionally for 6 months. After 6 months, renal artery angiography was performed again to observe the condition of the renal arteries, after which all animals were euthanized. The blood pressure and blood biochemical results of each group were evaluated 6 months after the operation; kidney tissue morphology and collagen
\end{abstract}

karger@karger.com www.karger.com/kbr

Karger!
(C) 2021 The Author(s)

Published by S. Karger AG, Basel

This is an Open Access article licensed under the Creative Commons Attribution-NonCommercial-4.0 International License (CC BY-NC) (http://www.karger.com/Services/OpenAccessLicense), applicable to the online version of the article only. Usage and distribution for commercial purposes requires written permission. fiber content were examined by hematoxylin-eosin staining and Masson staining; superoxide dismutase activity and the malondialdehyde content of kidney tissue were assessed by a biochemical enzyme method; the protein expression level of transforming growth factor- $\beta 1$ (TGF- $\beta 1$ ), a-smooth muscle actin ( $\alpha-S M A)$, and Smad 3 was assessed by Western blot, and electron microscopy was used to examine changes in the kidney microstructure. Results: After 6 months of a highfat diet, the blood lipid levels of the model group were significantly higher compared to baseline and to that of the control group during the same period (all showed $p<0.05$ ); the blood lipid levels of the control group did not change significantly from baseline $(p>0.05)$. The degree of glomerular damage caused by hyperlipidemia in the intima-RDN group and the adventitia-RDN group was significantly lower than that of the sham and control groups, and the renal fibrosis area percentage was also significantly lower $(p<0.05)$. Electron microscopy showed that both the intima-RDN group and the adventitia-RDN group had a more even distribution of chromosomes and less mitochondrial swelling compared with the sham group. Conclusion: RDN from the adventitia of the renal artery and RDN from the intima of the Binbin Zhu and Yahui Liu contributed equally to this work.
Correspondence to:

Chuanyu Gao, gaocy6208@163.com 
renal artery have the similar advantages of delaying high-fatinduced renal fibrosis. The antifibrotic effect of RDN may be related to inhibition of the TGF- $\beta 1 /$ Smad3 pathway.

(C) 2021 The Author(s).

Published by S. Karger AG, Basel

\section{Introduction}

Hypercholesterolemia not only plays an important role in the occurrence and progression of cardiovascular disease but also is closely related to chronic kidney disease and exacerbates the development of kidney disease [1]. In related experiments in rodents, it was found that the increase in oxidative stress related to hypercholesterolemia can cause glomerular capillary endothelial dysfunction and aggravate the development of glomerular sclerosis associated with lipid glomerular deposition [2]. Studies have also confirmed that hyperlipidemia induced by high cholesterol feeding can cause renal endothelial cell and microvascular dysfunction, resulting in inflammatory infiltrates and the progression of renal fibrosis [3].

In 2009, Krum et al. [4] used RDN for the first time to treat resistant hypertension. They accomplished this by using radiofrequency energy to destroy sympathetic nerve fibers around the renal artery, thereby reducing the patient's overall sympathetic activity as well as that of the kidney [4]. Related research studies have explored the potential therapeutic effects of RDN in heart failure, insulin resistance, obstructive sleep apnea syndrome, atrial fibrillation, and end-stage renal disease [5]. In addition, research on RDN in related anticardiac hypertrophy shows that its effect is related to antifibrosis [6]. However, there is no relevant research to explore whether RDN is able to slow down the progression of chronic renal fibrosis induced by high fat intake.

Conventional intravascular $\mathrm{RDN}$ requires heat energy to be transmitted through the vessel wall to the sympathetic nerve bundles surrounding the renal blood vessels, which may cause damage to the arterial endothelium, leading to atherosclerosis and luminal stenosis $[7,8]$. Some scholars have reported the use of the RDN method on the lateral renal artery [9], confirming that sympathetic nerve ablation is safe and effective. The present study aims to compare whether 2 different routes of RDN (from the intima and adventitia of the renal artery) can reduce renal fibrosis in a pig model of hypertension induced by a high-fat diet and to explore possible molecular mechanisms.

\section{Materials and Methods}

\section{Animals}

Twenty-four male Bama pigs ( 8 months old, $22.42 \pm 0.78 \mathrm{~kg}$ ) were purchased from Beijing Strong Century Mini-pigs Breeding Base (Beijing, China, Approval No. SYXK (jing) 2018-0040). All pigs were maintained at a controlled temperature and humidity on a 12:12-h dark-light cycle. Twenty-four Bama swine were randomly assigned to 2 groups: the control group $(n=6)$ or the high-fat diet group ( $n=18)$; the high-fat diet group was further randomly divided into the intima-RDN group $(n=6)$, the adventitia-RDN group $(n=6)$, or the sham group $(n=6)$. All animals were fed separately. A high-fat $(4,100 \mathrm{kcal} / \mathrm{kg})$ diet consisting of protein (10\%), fat (41\%), carbohydrates (43\%), and minerals (6\%) was initiated on the first day after RDN or renal arteriography. The daily high-fat diet intake was approximately $5 \%$ of the body weight of each animal and was adjusted dynamically every 2 weeks. All experimental procedures were approved by the Animal Care Ethics Committee of Zhengzhou University (ZZU-LAC20180518[09]) and were conducted in accordance with the American Physiology Society's "Guides for the Care and Use of Laboratory Animals" published by the National Institutes of Health (NIH Publication No. 85-23, revised 1996).

Before the operations, animals were provided a liquid diet for 2 days, followed by fasting for $12 \mathrm{~h}$ with free access to water. Venous blood was collected for measurements of renal function and lipid levels while pigs were under general anesthesia. Anticoagulant therapy was administered with an initial unfractionated heparin bolus (100 IU/kg IV), followed with 1,000 IU each additional hour. Renal arterial angiography was performed to record the images of both renal arteries before the operation. Each mini-pig in the intima-RDN group $(n=6)$ underwent bilateral catheter-based $\mathrm{RDN}$ from the intima of the renal artery; each mini-pig in the adventitia-RDN group $(n=6)$ underwent bilateral laparoscopybased RDN from the adventitia of the renal artery; every mini-pig in the sham group $(n=6)$ underwent the same procedure except with radiofrequency ablation. Renal arterial angiography was performed again for follow-up before all swine were killed. In the end, all pigs were heparinized and euthanized under deep anesthesia. The kidneys were removed and stored in liquid nitrogen, and the renal arteries were removed and stored in formalin and liquid nitrogen for further analysis.

\section{Anesthesia and Surgical Technique}

The pigs were sedated by administering an intramuscular injection of ketamine hydrochloride $(5 \mathrm{mg} / \mathrm{kg})$, midazolam $(0.5 \mathrm{mg} /$ $\mathrm{kg})$, and atropine $(0.5 \mathrm{mg})$ to induce anesthesia, and an infusion channel was established by piercing the edge of the vein. Intravenous supplemental propofol $(3 \mathrm{mg} / \mathrm{kg})$, sufentanil $(1 \mathrm{~g} / \mathrm{kg})$, and vecuronium bromide $(0.1 \mathrm{mg} / \mathrm{kg})$ were administered prior to endotracheal intubation and before being connected to the anesthesia machine. Sevoflurane $(0.5-1.5 \mathrm{mac})$ was inhaled throughout the operation; its flow was controlled through an anesthesia ventilator (Dräger Fabius, Lübeck, Germany). The ventilator parameters were set as follows: tidal volume $12-15 \mathrm{~mL} / \mathrm{kg}$, respiratory rate 16-18 breaths/min, and a suction/inhalation ratio of 1:2 to maintain anesthesia. This technique was used for anesthesia and monitored by a senior anesthesiologist. During the operation, blood oxygen saturation was closely monitored, and venous blood was drawn for routine biochemical testing. 
For the intima-RDN group, the femoral arteries were punctured under anesthesia by the vascular incision method. A 7F sheath (Cordis Corporation, Hialeah, FL, USA) was introduced into the artery and secured. Each pig was heparinized at $100 \mathrm{U} / \mathrm{kg}$. An angiographic catheter (Cordis Corporation) was inserted into the abdominal aortic region at the origin of the renal artery. The angiographic catheter was withdrawn, followed by insertion of a temperature-controlled cardiac radiofrequency (RF) catheter (NS7TCDL174HS; Biosense Webster, Diamond Bar, CA, USA), which was connected to a generator (Johnson \& Johnson, New Brunswick, NJ, USA). For the adventitia-RDN group, pigs were placed in the lateral position and secured with straps. The first incision was made approximately $2 \mathrm{~cm}$ below the intersection of the posterior axillary line and rib margin, and pneumoperitoneum was achieved with air insufflation. The second incision was made approximately $2 \mathrm{~cm}$ above the intersection of the midaxillary line and spina iliaca. The third incision was made symmetric to the first incision in the anterior axillary line. A $10-\mathrm{mm}$ trocar was then inserted into the second incision. Subsequently, two 5-mm trocars were inserted into the first and third incisions. A celioscope lens was introduced through the $10-\mathrm{mm}$ trocar, and 2 graspers were introduced through the two 5-mm trocars. After the selected renal artery was exposed and separated by the 2 graspers, a 7-Fr diameter RF ablation catheter (Biosense Webster), which was connected to an RF generator (Biosense Webster), was introduced into the retroperitoneum through a $5-\mathrm{mm}$ trocar and carried by using a grasper. These steps were the same for the intima-RDN group.

Both the intima-RDN group and the adventitia-RDN group underwent the following radiofrequency ablation procedures: $8 \mathrm{~W}$ or less lasting up to $120 \mathrm{~s}$ each to obtain up to 6 ablations, separated both longitudinally and rotationally from the adventitia of the renal arteries. A saline solution was injected into the top of the RF catheter to control the temperature. After the procedure, the RF catheter was removed, and angiography was performed again to check the anatomical structure of the renal arteries. The same procedure, except for the ablation, was performed on animals in the sham group. This technique was performed by experienced urologists.

\section{Histopathological Examination of the Renal Cortex}

The renal cortex was collected and evaluated by histological staining. Each renal cortex was fixed for $24 \mathrm{~h}$ in $4 \%$ paraformaldehyde. After fixation, the tissues were washed, then dehydrated by soaking in a concentration gradient $(75,85,90,95$, and $100 \%)$ of alcohol, and, lastly, cleared in xylene. Next, the tissues were embedded in paraffin wax. The samples were then subjected to HE and Masson staining.

\section{Measurements of BP and Blood Biochemistry Test}

BP was measured using a BP-2010E monitor (Softron, Beijing, China) at baseline and at 6 months after surgery. Serum creatinine (Scr), total cholesterol (TC), and triglyceride (TG) levels were assessed using an automatic biochemical analyzer (Rayto, Shenzhen, China). The concentration of norepinephrine (NE) in the renal tissue was assessed using an ELISA kit (USCN Business Co. Ltd., Wuhan, China).

Western Blot Detection of TGF- $\beta 1, \alpha-S M A$, and Smad3 Expression

For protein expression analysis, the renal cortex was washed 2-3 times with cold PBS and homogenized by liquid nitrogen mill- ing. The supernatant protein concentration was measured using a BCA Protein Assay Kit (G2026; Servicebio, Wuhan, China). The protein was separated on an $8-10 \%$ gel by SDS-PAGE and transferred to PVDF membranes at a constant current of $300 \mathrm{~mA}$ for half an hour. The membranes were then blocked for an hour with $5 \%$ skim milk dissolved in $0.5 \%$ TBST. Subsequently, the membranes were incubated at $4^{\circ} \mathrm{C}$ overnight with primary rabbit polyclonal anti-endothelial nitric oxide synthase (eNOS; 1:1,000; ab5589; Abcam, Cambridge, UK), rabbit polyclonal anti-phosphorylated eNOS Ser1177 (1:1,000; 9571; CST, Boston, MA, USA), rabbit polyclonal anti-acetophenone (AKT; 1:1,000; 9272; CST), rabbit polyclonal anti-phosphorylated AKT Ser473 (1:1,000; 9271; CST), and mouse monoclonal anti-glyceraldehyde phosphate dehydrogenase (GAPDH; 1:25,000; GB13002-m-1; Servicebio) antibodies. The membranes were washed 3 times with TBST buffer at room temperature and then incubated with peroxidase-labeled goat anti-rabbit IgG $(\mathrm{H}+\mathrm{L})$ secondary antibody $(1: 3,000$; 0741506; KPL, Gaithersburg, MD, USA) and peroxidase-labeled goat anti-mouse IgG $(\mathrm{H}+\mathrm{L})$ secondary antibody (1:3,000; 074-1806; KPL) for half an hour. AlphaEaseFC software (Alpha Innotech, San Jose, CA, USA) was used to analyze the optical density of the target bands.

\section{Statistical Analysis}

All statistical analyses were performed with SPSS 22.0 software (SPSS Inc., Chicago, IL, USA). Continuous data are presented as the mean \pm standard deviation (SD). The normality of the distribution was assessed with the Shapiro-Wilk test. Variables with normal distributions were compared using the ANOVA test, whereas variables with skewed distributions were compared with the Mann-Whitney U test. $p$ values $<0.05$ were considered statistically significant.

\section{Results}

\section{Renal Artery Angiography 6 Months after Surgery in} the RDN Group

Comparing the renal artery angiography of the $2 \mathrm{RDN}$ groups of mini-pigs before and 6 months after RDN revealed that no obvious renal artery stenosis, plaque, deformity, or other abnormalities were observed in either the intima-RDN group or the adventitia-RDN group (Fig. 1).

\section{Serum Creatinine Levels and Lipid Profiles at Baseline and after 6 Months}

Compared with baseline, significant increases in TC, TG, and LDL-C and a significant decrease in HDL-C were observed in pigs in the sham group, intima-RDN group, and adventitia-RDN group (all $p<0.05$ ) after a 6-month high-fat diet. However, the differences in Scr, TC, TG, HDL-C, and LDL-C between the RDN groups and sham group after 6 months were not significant (Table 1). 


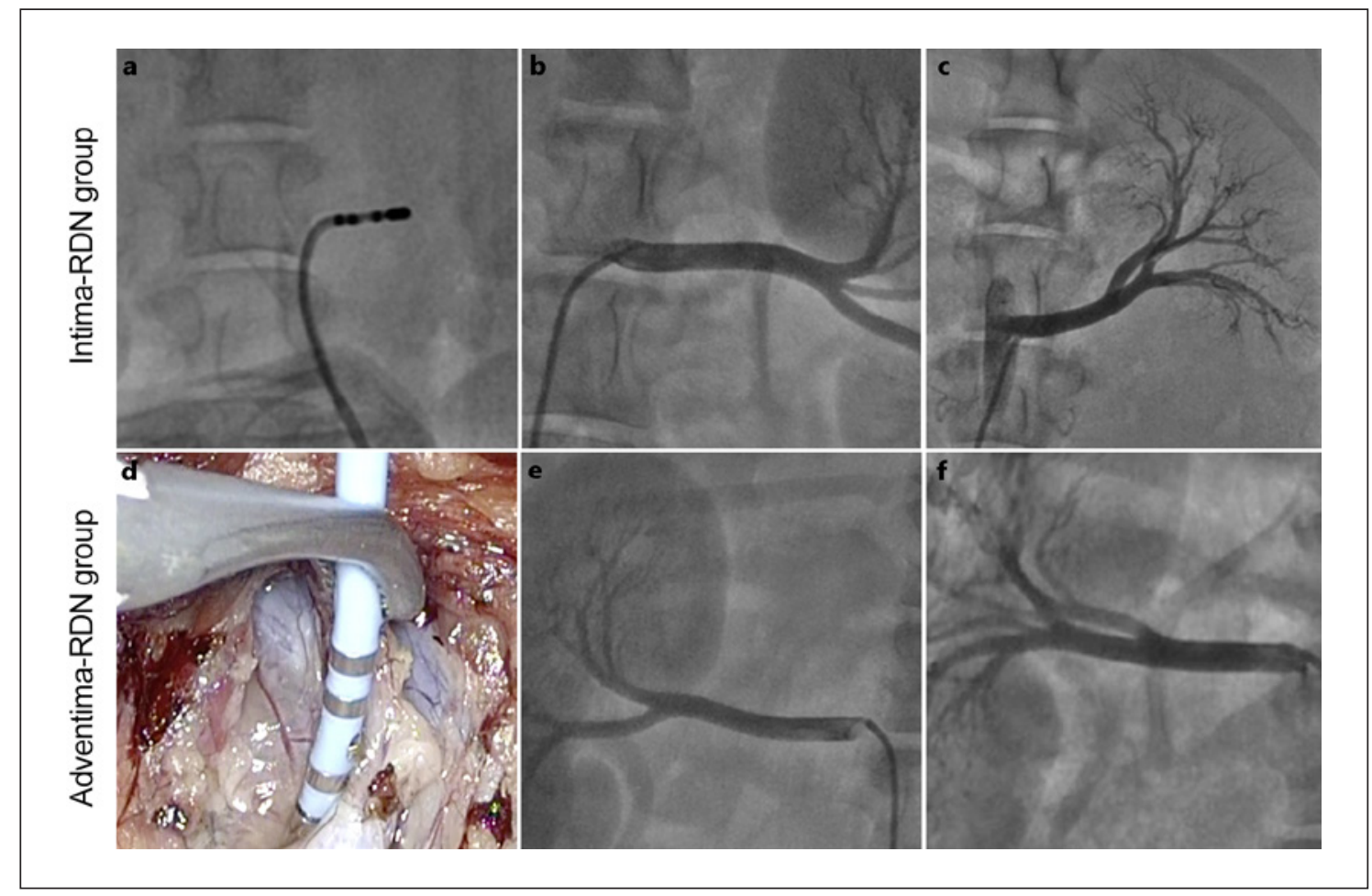

Fig. 1. Intima-RDN group and adventitia-RDN group Bama miniature pigs undergoing renal artery angiography. Renal artery radiofrequency ablation in the intima-RDN group (a); preoperative renal artery angiography in the intima-RDN group (b); postoperative renal artery angiography in the intima-RDN group (c); RDN from the adventitia of the renal artery (d); preoperative renal artery angiography in the adventitia-RDN group (e); postoperative renal artery angiography in the adventitia-RDN group (f). $n=6$ for each group. RDN, renal denervation.

Table 1. Levels of lipid profiles

\begin{tabular}{|c|c|c|c|c|c|c|c|c|}
\hline \multirow[t]{2}{*}{ Group } & \multicolumn{4}{|l|}{ Baseline } & \multicolumn{4}{|c|}{ After 6 months } \\
\hline & $\begin{array}{l}\text { intima-RDN } \\
\text { group }\end{array}$ & $\begin{array}{l}\text { adventitia-RDN } \\
\text { group }\end{array}$ & sham group & $\begin{array}{l}\text { control } \\
\text { group }\end{array}$ & $\begin{array}{l}\text { intima-RDN } \\
\text { group }\end{array}$ & $\begin{array}{l}\text { adventitia-RDN } \\
\text { group }\end{array}$ & sham group & $\begin{array}{l}\text { control } \\
\text { group }\end{array}$ \\
\hline $\mathrm{TC}(\mathrm{mmol} / \mathrm{L})$ & $2.57 \pm 0.43$ & $2.62 \pm 0.82$ & $2.59 \pm 0.61$ & $2.27 \pm 0.50$ & $3.53 \pm 0.47^{\#, *}$ & $3.46 \pm 0.95^{\#, *}$ & $3.54 \pm 0.55^{\#, *}$ & $2.07 \pm 0.35^{+}$ \\
\hline $\mathrm{TG}(\mathrm{mmol} / \mathrm{L})$ & $1.05 \pm 0.27$ & $1.11 \pm 0.41$ & $1.16 \pm 0.32$ & $1.08 \pm 0.19$ & $1.73 \pm 0.50^{\#}$ & $1.72 \pm 0.37^{\#}$ & $1.75 \pm 0.33^{\#}$ & $1.32 \pm 0.19$ \\
\hline $\mathrm{HDL}-\mathrm{C}(\mathrm{mmol} / \mathrm{L})$ & $1.79 \pm 0.49$ & $1.61 \pm 0.12$ & $1.77 \pm 0.15$ & $1.68 \pm 0.35$ & $0.85 \pm 0.23^{\#, *}$ & $0.93 \pm 0.47^{\#, *}$ & $0.84 \pm 0.10^{\#, *}$ & $1.56 \pm 0.22^{+}$ \\
\hline LDL-C (mmol/L) & $2.11 \pm 0.40$ & $2.12 \pm 0.67$ & $2.12 \pm 0.40$ & $2.04 \pm 0.32$ & $3.88 \pm 0.95^{\#, *}$ & $4.04 \pm 0.73^{\#, *}$ & $4.09 \pm 0.71^{\#, *}$ & $2.22 \pm 0.29^{+}$ \\
\hline $\mathrm{SCr}(\mu \mathrm{mol} / \mathrm{L})$ & $84.89 \pm 13.30$ & $82.41 \pm 3.30$ & $83.30 \pm 7.19$ & $84.27 \pm 10.46$ & $84.30 \pm 5.71$ & $79.96 \pm 10.25$ & $83.99 \pm 8.78$ & $81.48 \pm 6.01$ \\
\hline
\end{tabular}

Data are expressed as the mean \pm standard deviation. $n=6$ per group. RDN, renal denervation; TC, total cholesterol; TG, triglyceride; HDL-C, high-density lipoprotein cholesterol; LDL-C, low-density lipoprotein cholesterol. ${ }^{*} p<0.05$ versus baseline of the same group. ${ }^{*} p<0.01$ versus the control group at the same time point. ${ }^{+} p<0.01$ versus the sham group at the same time point.

\section{Changes in Blood Pressure at Baseline and after 6 \\ Months}

The baseline systolic blood pressures (SBP) of the control group, sham group, intima-RDN group, and adventitia-RDN group were $124.17 \pm 2.93 \mathrm{~mm} \mathrm{Hg}$,
$124.17 \pm 2.93 \mathrm{~mm} \mathrm{Hg}, 123.33 \pm 4.23 \mathrm{~mm} \mathrm{Hg}$, and 122.67 $\pm 3.20 \mathrm{~mm} \mathrm{Hg}$, respectively. There was no significant difference between the groups. The baseline diastolic blood pressures (DBP) in the control group, sham group, intima-RDN group, and adventitia-RDN group 


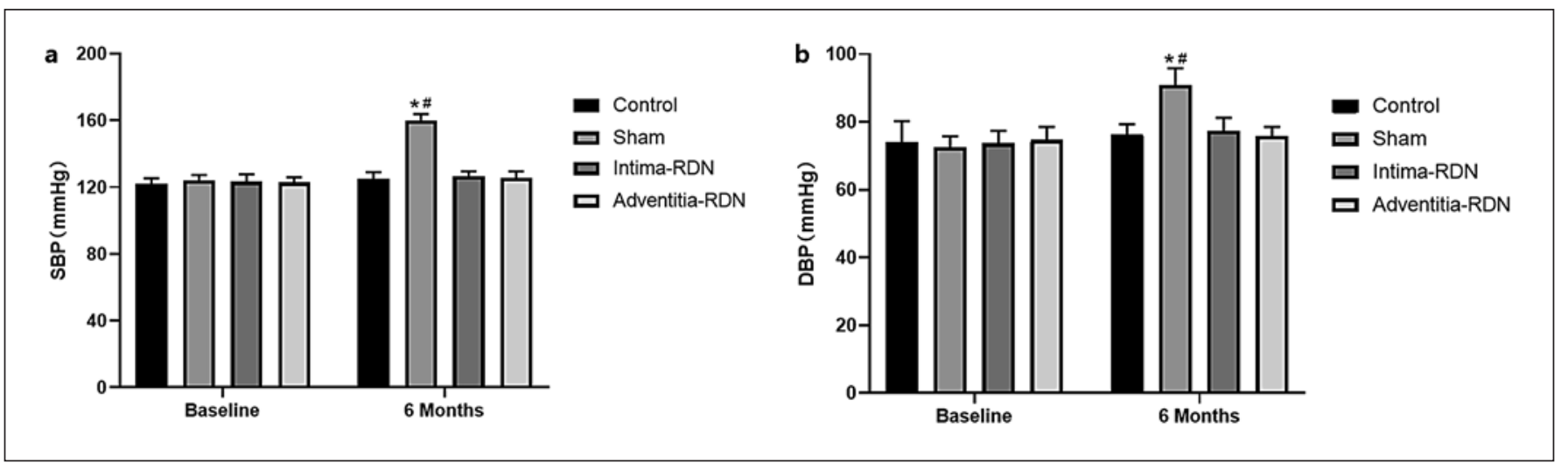

Fig. 2. Changes in blood pressure in the 4 groups at baseline and after 6 months. Changes in SBP at baseline and after 6 months (a); changes in DBP at baseline and after 6 months (b). SBP, systolic blood pressure; DBP, diastolic blood pressure. ${ }^{*} p<0.05$ compared with baseline values in the sham group. Data are presented as mean \pm standard deviation ( $n=6$ for each group). ${ }^{\#} p<0.05$ versus the sham group after 6 months.

were $74.00 \pm 6.26 \mathrm{~mm} \mathrm{Hg}, 72.50 \pm 3.28 \mathrm{~mm} \mathrm{Hg}, 73.67$ $\pm 3.67 \mathrm{~mm} \mathrm{Hg}$, and $74.67 \pm 3.83 \mathrm{~mm} \mathrm{Hg}$, respectively. After the 6-month high-fat diet, the SBP of the control group $(121.83 \pm 3.37 \mathrm{~mm} \mathrm{Hg})$, intima-RDN group $(126.67 \pm 2.73 \mathrm{~mm} \mathrm{Hg})$, and adventitia-RDN group $(125.50 \pm 3.94 \mathrm{~mm} \mathrm{Hg})$ were all significantly lower than that of the sham group $(160.00 \pm 3.74 \mathrm{~mm} \mathrm{Hg}$, all $p<$ $0.001)$. The DBP of the control group $(76.33 \pm 3.01 \mathrm{~mm}$ $\mathrm{Hg})$, intima-RDN group $(77.33 \pm 3.88 \mathrm{~mm} \mathrm{Hg})$, and adventitia-RDN group $(75.83 \pm 2.71 \mathrm{~mm} \mathrm{Hg})$ were all significantly lower than that of the sham group (90.83 \pm $5.00 \mathrm{~mm} \mathrm{Hg}$, all $p<0.001)$. However, the SBP and DBP of the intima-RDN group and the adventitia-RDN group were not significantly different from those of the control group (all $p>0.05$, Fig. 2).

\section{Pathological Evaluation of the Kidney Tissue}

Kidney tissue hematoxylin and eosin staining (Fig. 3) showed that there was no obvious glomerular inflammation in the control group (Fig. 3a) and showed necrosis and expansion of renal tubular epithelial cells (Fig. 3e). Compared with the sham group, the glomerular inflammation (Fig. 3b-d) as well as the necrosis and expansion of renal tubular epithelial cells (Fig. 3f-h) in the intima and adventitia RDN groups were significantly reduced. Masson staining showed that there were no obvious renal interstitial fibers in the control group. Compared with the sham operation group, the renal interstitial fibrosis in the intima-RDN group and the adventitia-RDN group was significantly reduced (Fig. 3j-1). Image-Pro Plus 6.0 software was used to quantify the renal fibrosis area percentage.

Renal Interstitial Fibrosis Is Reduced in

Pigs after Renal Denervation
Use of a Biochemical Enzyme Method to Detect SOD Activity and MDA Content in Kidney Tissue

The content of the lipid oxidation metabolite MDA in the sham group $(4.50 \pm 1.40 \mathrm{nmol} / \mathrm{mL})$ and the RDN group $(3.02 \pm 0.57 \mathrm{nmol} / \mathrm{mL}$ in the intima-RDN group and $2.64 \pm 0.64 \mathrm{nmol} / \mathrm{mL}$ in the adventitia-RDN group) was higher than that in the control group $(1.12 \pm 0.22$ $\mathrm{nmol} / \mathrm{mL}, p<0.001)$. However, the intima-RDN group and the adventitia-RDN group were both significantly lower than the sham operation group $(p=0.037$ and $p=$ 0.014 , respectively). In addition, the content of plasma antioxidant SOD in the control group (196.14 $\pm 16.16 \mathrm{U} /$ $\mathrm{mL}$ ) was significantly higher than that of the intimaRDN group $(168.38 \pm 13.05 \mathrm{U} / \mathrm{mL}, p=0.008)$, adventitiaRDN group $(178.84 \pm 13.32 \mathrm{U} / \mathrm{mL})$, and sham group $(116.72 \pm 14.99 \mathrm{U} / \mathrm{mL}, p<0.001)$. The SOD content in the intima-RDN group and adventitia-RDN group was significantly higher than that in the sham group (all $p<$ 0.001, Fig. 4).

Protein Expression Levels of TGF- $\beta 1, \alpha-S M A$, Smad3, and NE Concentration in the Renal Tissue

Six months after surgery, the expression of TGF- $\beta 1$, $\alpha$-SMA, and Smad3 proteins was significantly different between the RDN and sham groups. Significantly lower TGF- $\beta 1$ and Smad 3 expression was observed in the RDN groups than in the sham group at 6 months after surgery (Fig. 5). The NE concentrations in renal tissues in the control group, intima-RDN group, and adventitia-RDN group were significantly lower than those in the sham group at 6 months after surgery $(134.35 \pm 13.56 \mathrm{ng} / \mathrm{g}$ vs. 


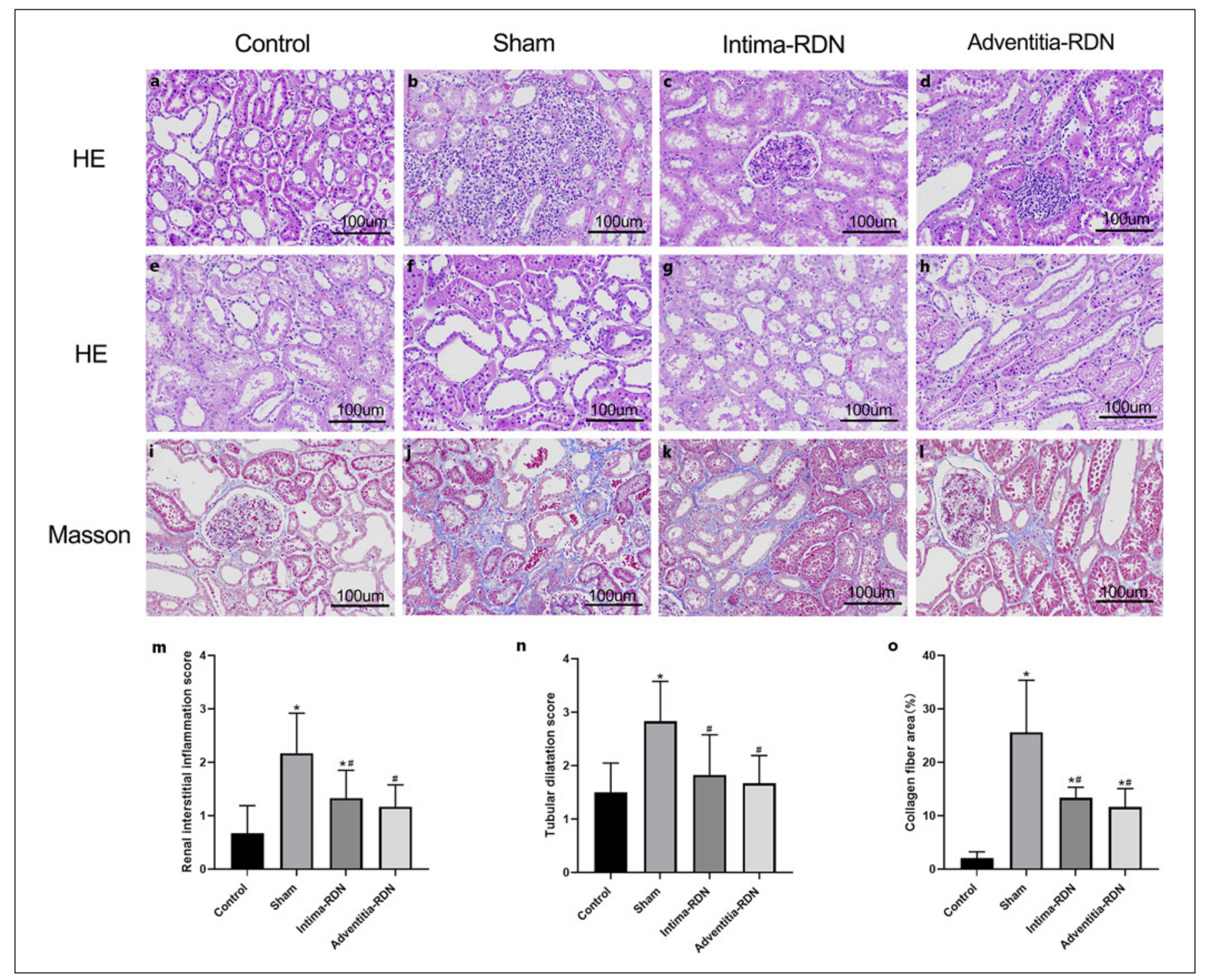

Fig. 3. Renal tubules and interstitial pathology after 6 months in each group. In the control group, there was no obvious glomerular inflammation (HE. $\times 200)(\mathbf{a})$; necrosis and the expansion of renal tubular epithelial cells $($ HE. $\times 200)(\mathbf{e})$; compared with the sham operation group, glomerular inflammation in the intimal and adventitia RDN groups was significantly reduced $($ HE. $\times 200)(\mathbf{b}-\mathbf{d})$; compared with the sham operation group, necrosis and the expansion of renal tubular epithelial cells in the intimal and adventitia
RDN groups were significantly reduced (HE. ×200) (f-h); Masson staining showed that there was no obvious renal interstitial fibrosis in the control group (Masson. $\times 200)(\mathbf{i})$; compared with the sham operation group, endometrium-renal interstitial fibrosis was significantly reduced in the intima-RDN group and the adventitiaRDN group (Masson. $\times 200)(\mathbf{j}-\mathbf{I}) . n=6$ for each group. Renal interstitial inflammation score in four groups $(\mathbf{m})$; tubular dilatation score in four group (n); collagen fiber area in four groups (o).
$342.33 \pm 18.02 \mathrm{ng} / \mathrm{g}, 200.57 \pm 19.33 \mathrm{ng} / \mathrm{g}$ vs. $342.33 \pm$ $18.02 \mathrm{ng} / \mathrm{g}$, and $175.51 \pm 16.47 \mathrm{ng} / \mathrm{g}$ vs. $42.33 \pm 18.02$, all $p<0.001$, Fig. 5).

\section{Electron Microscopic Analysis}

Microstructural changes in the kidneys were observed with an electron microscope (Fig. 6). In the control group, podocyte chromatin distribution is relatively uniform, heterochromatin is diminished, mitochondria are not significantly swollen, and the basement membrane is not thickened (Fig. 6a). In the sham group, podocyte nuclear heteromorphism is present, with significantly increased heterochromatin, swelling of mitochondria, lack of cristae, severe endoplasmic reticulum expansion, and au- 


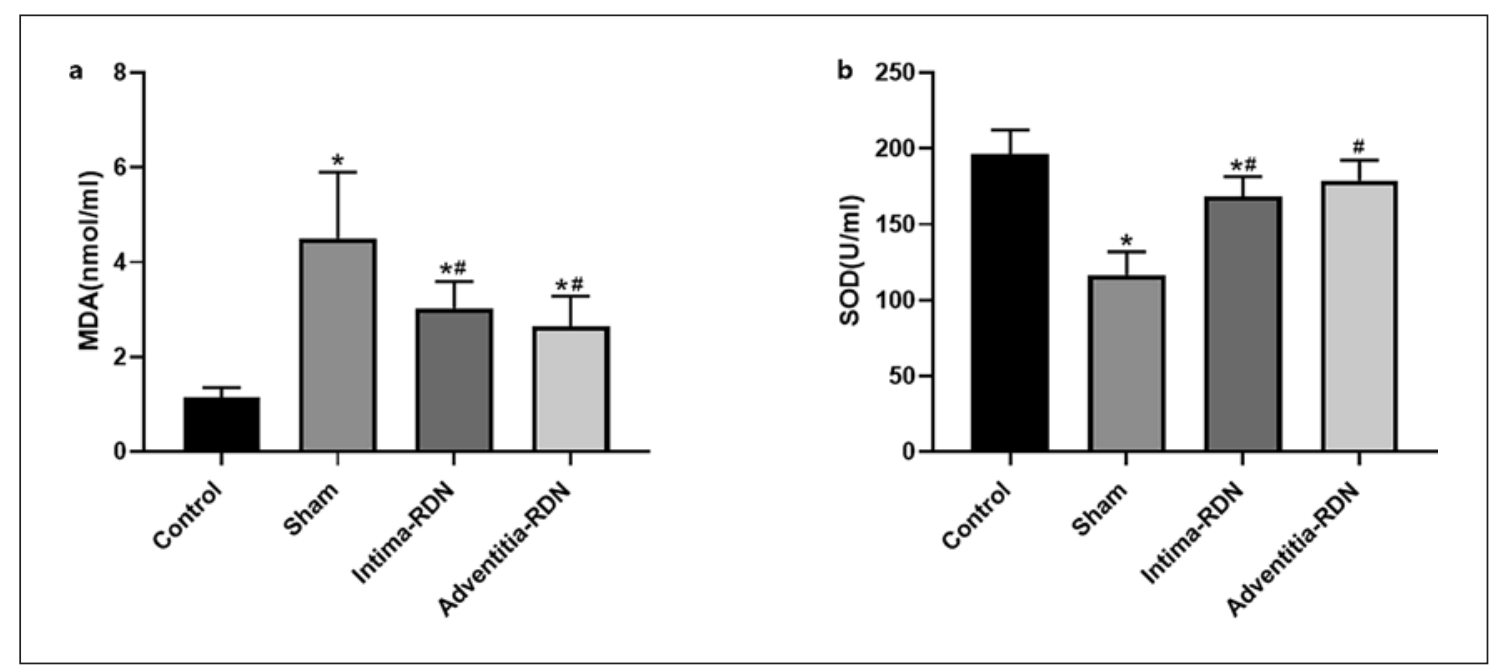

Fig. 4. a, b The effects of intima-RDN and adventitia-RDN on serum T-SOD and MDA levels. ${ }^{*} p<0.05$ versus the control group. ${ }^{\#} p<0.05$ versus the sham group. Data are presented as mean \pm standard deviation $(n=6$ for each group). T-SOD, total superoxide dismutase; MDA, malondialdehyde.

tophagosomes, as well as uneven basement membrane thickness with some obvious thickening (Fig. 6b). In the intima-RDN group, podocytes are stained frequently, are more evenly distributed and with less heterochromatin, mitochondria are slightly swollen, and basement membrane thickness is uneven with some thickening (Fig. 6c). In the adventitia-RDN group, basement membrane thickness is slightly uneven, and some areas are fuzzy (Fig. 6d).

\section{Discussion}

The main findings of this study were that (1) RDN is able to reduce BP in a pig model of hypertension induced by a high-fat diet, (2) RDN is also able to slow down the progression of high fat-induced kidney damage and changes in kidney structure, and (3) RDN from the adventitia of the renal artery and RDN from the intima of the renal artery have the same advantages of delaying high fatinduced renal fibrosis. Lipotoxicity can occur in several organs, such as the liver, heart, and skeletal muscle. Research has shown that the kidney is vulnerable to lipotoxicity and undergoes highly relevant metabolic alterations, which may induce cell death and inflammation [10]. Excessive intake of high-calorie and high-cholesterol-containing foods can also increase blood pressure [11], which may eventually lead to kidney disease. This process is related to mechanisms that include inflammation, oxidative stress, and sympathetic overactivation [12]. The increase in oxidative stress induced by a high-cholesterol diet is closely related to organ fibers [13] because hypercholesterolemia can cause vascular endothelial cell dysfunction, trigger the activation of inflammatory factors, and enhance oxidative stress and inflammatory infiltration of target organs, thereby exacerbating renal damage and the process of chronic renal fibrosis $[14,15]$. Fibrosis is a common pathway for organ damage and failure. Therefore, it is extremely important to find an effective treatment to slow down, avoid, or even reverse the progression of tissue fibrosis. Patients with chronic and end-stage renal failure usually also have hypertension [16], which may be related to high blood volume, renin-angiotensin-aldosterone system (RAAS) activation, and sympathetic excitation. RDN surgery, which has emerged in recent years, achieves the purpose of lowering blood pressure by blocking the activity of the renal sympathetic nervous system (SNS) and inhibiting the activity of the RAAS.

In recent years, RDN surgery has achieved the goal of lowering BP by blocking the activity of the renal SNSs and inhibiting the activity of RAAS. RDN has been shown to be effective in reducing BP in a variety of hypertensive animals, including dogs, pigs, and sheep, as well as SHRs [17-19]. Based on the abovementioned basis, RDN may have potential benefits for chronic kidney disease [20, $21]$. The results of many previous studies have shown that high-fat-induced intake can lead to chronic kidney lesions or even significantly aggravated fibrosis development in kidneys of rats [22-25]. Moreover, studies have 


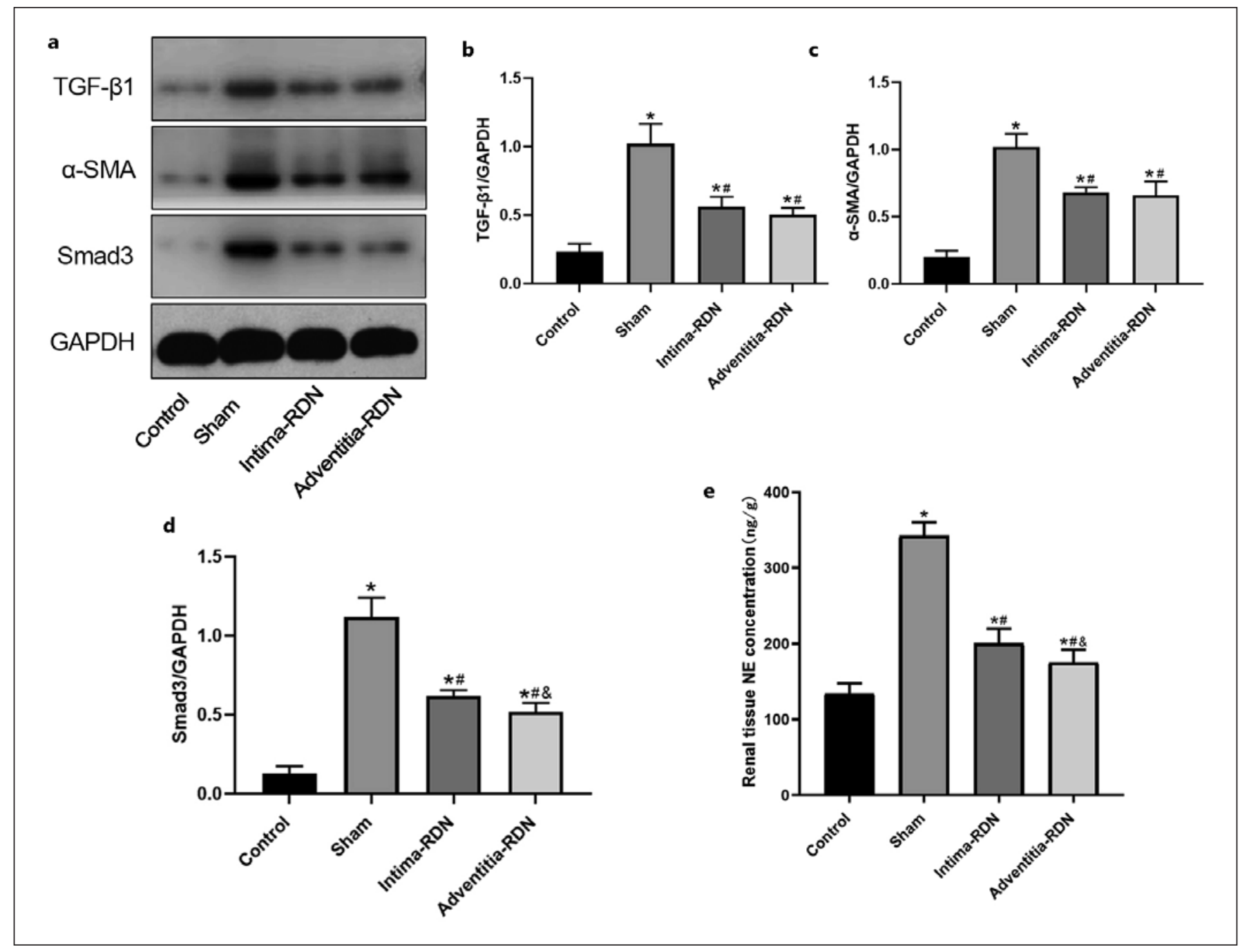

Fig. 5. Changes in TGF- $\beta 1, \alpha-S M A$, Smad3, and NE levels at 6 months. a Representative images of Western blots of TGF- $\beta 1, \alpha-S M A$, and Smad3 protein expression and the corresponding quantitation in the control, sham, and RDN groups (b-d); changes in renal NE at 6 months $(\mathbf{e})$. Data are presented as mean \pm standard deviation $(n=$ 6 for each group). ${ }^{*} p<0.05$ compared with the control group, ${ }^{*} p<0.05$ compared with the sham group, ${ }^{8} p<0.05$ compared with the intima-RDN group.

also shown that RDN can effectively reduce the BP of hypertensive dogs caused by obesity, as well as renal pathological changes caused by hypertension [26].

In traditional catheter-based RDN from the intima of the renal artery, radiofrequency ablation energy directly acts on the intima of renal blood vessels, which inevitably causes damage to renal vascular endothelial function; such vascular endothelial injury is the initiating factor for atherosclerosis. Relevant studies have shown that a series of surgery-related complications, such as renal vascular injury, renal artery stenosis, edema at the ablation site, and thrombosis, may occur at different times after RDN
[27-29]. Moreover, combined with the anatomical characteristics of the kidney, renal artery sympathetic nerve fibers are mostly distributed on the surface of the adventitia of the renal artery [30]. Traditional RDN from the intima of the renal artery may not be able to completely ablate the distal end of the renal artery and its branch vessels. For this reason, our team innovated a new way of implementing RDN through the adventitia of the renal artery. In this study, we compared the effects of 2 different $\mathrm{RDN}$ pathways on high-fat-induced renal fibrosis.

The results showed that the blood lipid levels of each group except the control group were significantly higher 

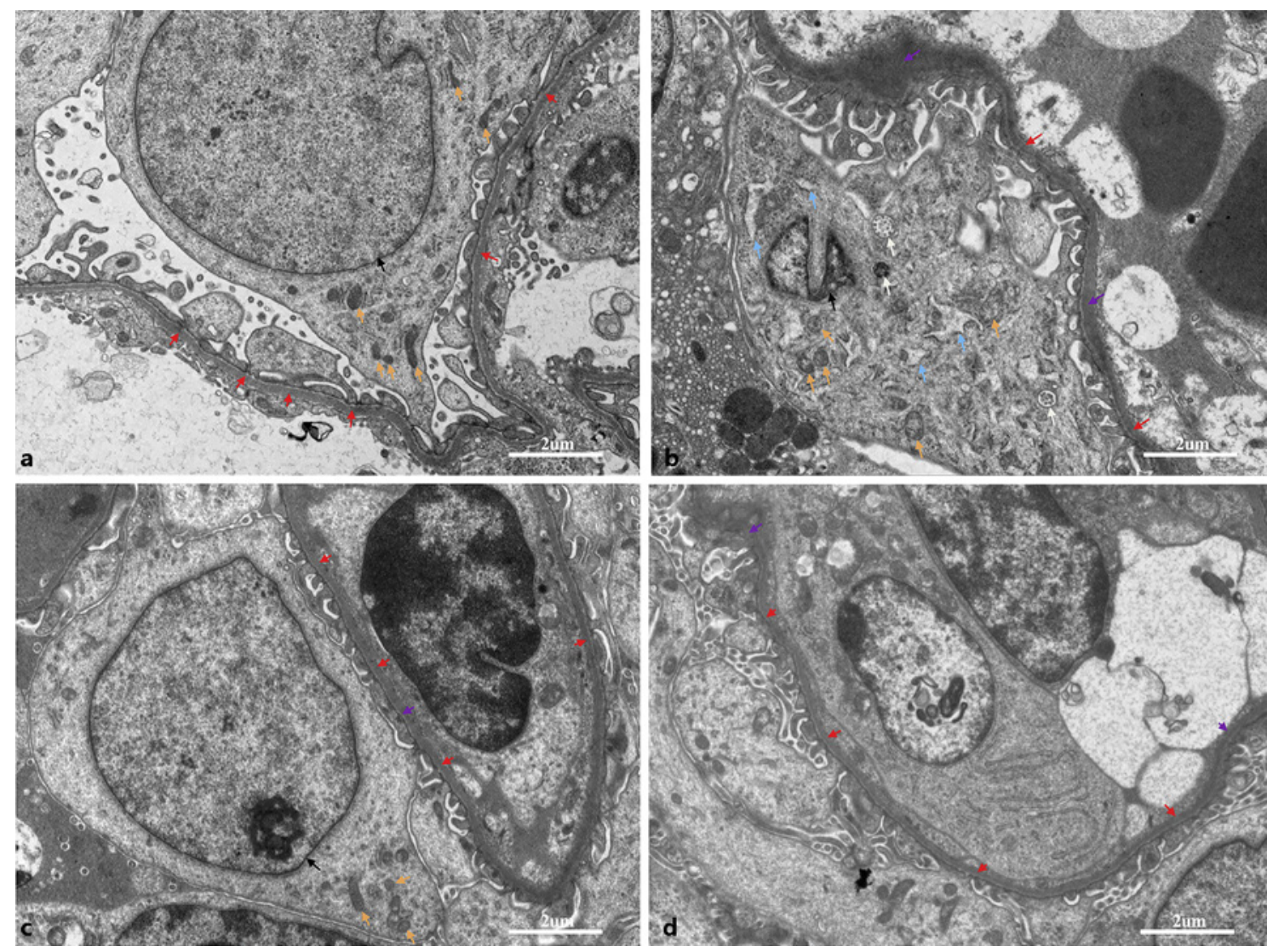

Fig. 6. Electron microscopy of renal tissue $(\times 2,500)$ in each group 6 months after surgery. Control group: podocytes with more uniform chromatin distribution and less heterochromatin (black), no obvious swelling of mitochondria (orange), and no significant increase in basement membrane thickness (red) (a); sham operation group: podocyte nuclear atypia with significantly increased heterochromatin (black); swelling of mitochondria, a lack of cristae (orange), and severe endoplasmic reticulum expansion (blue); au- tophagic bodies present (white); basement membrane thickness is uneven (red), with a part obviously thickened (purple) (b); intimaRDN group: podocytes stain often more evenly distributed and less heterochromatin (black); mitochondria are slightly swollen (orange); basement membrane thickness is uneven (red), and partly thickened (purple) (c); and adventitia-RDN group: basement membrane thickness is slightly uneven (red) and partly blurred (purple) (d). than the baseline at 6 months after operation, and the BP level of RDN groups was similar to that of the control group, which was significantly lower than that of the sham group. The above results suggested that the hypertension model induced by high-fat diet was successful. Furthermore, Scr levels were similar at 6 months after surgery among the 4 groups, suggesting that RDN does not affect glomerular function, which is supported by previous studies [31, 32].

$\mathrm{NE}$ is the main catecholamine neurotransmitter in the SNS and an important indicator of sympathetic nerve function. In this study, the level of NE in renal tissue in the $\mathrm{RDN}$ groups was significantly lower than that in the sham group. Furthermore, the magnitude of decrease was greater in the adventitia-RDN group than that in the intima-
RDN group, which is consistent with our team's previous research results involving beagle dogs. The above results demonstrate that RDN is capable of destroying the function of the renal artery sympathetic nerve bundle and inhibiting the overactivated SNS; adventitia-RDN is able to achieve better inhibition of the sympathetic nervous system than intima-RDN. Moreover, compared with the intima-RDN group, there was no vasospasm, edema, or thrombosis in the adventitia-RDN group [33]. The present study also showed that the SBP and DBP of the adventitia-RDN group and the intima-RDN group were significantly lower than those of the sham group, and the SBP and $\mathrm{DBP}$ of the adventitia-RDN group decreased more than that of the intima-RDN group, further indicating that RDN lowers blood pressure.
Renal Interstitial Fibrosis Is Reduced in Pigs after Renal Denervation
Kidney Blood Press Res 2022;47:135-146 DOI: $10.1159 / 000521100$ 
Oxidative stress results from an imbalance of the antioxidant and oxidant systems, which leads to excessive ROS production. Nucleoside phosphoric acid (NADPH) $\mathrm{p} 47^{\text {phox }}$ subunit consists of 390 amino acids, as a connector between the membrane and cytoplasmic components and necessary for NADPH oxidase activation and interstitial fibrosis $[34,35]$. The biological effect of NADPH oxidase is adjusted by antioxidant enzymes such as SOD and catalase. MDA, the lipid peroxidate product, is used as a measure of the body's oxidative levels. SOD is a vital antioxidant enzyme that effectively clears free oxygen radicals [36]. In our study, we found that RDN reduced the high level of oxidative stress after high-fat induction. In addition, the antioxidative stress ability of the RDN group was higher than that of the sham group.

Six months after RDN, renal artery angiography did not reveal any renal artery stenosis, plaque, deformity, or other abnormalities. According to the results of histopathological and electron microscopic, our study had shown that RDN could ameliorate glomerular lesions and renal tubulointerstitial fibrosis in hypertension, which has been verified in previous studies [37]. In contrast, our study introduced a new RDN pathway through the adventitia of the renal artery, and it had the similar effect on delaying the development of renal diseases comparing with the conventional RDN pathway through the intima of the renal artery, which has not been studied before.

Fibroblasts are static cells that maintain kidney structural integrity by producing a substantial level of extracellular matrix (ECM) in the renal interstitial. In the process of fibrosis, abnormal activation of fibroblast and expression of $\alpha$-SMA suggest that myofibroblasts play an important role in the pathogenesis of renal fibrosis. However, under certain conditions, fibroblasts may transform to myofibroblasts and participate in the process of renal fibrosis [38].

A large number of studies have confirmed that TGF- $\beta 1$ is a key cytokine that induces the transformation of renal fibroblasts into myofibroblasts and promotes fibrosis in renal diseases [39]. Smad3 protein is a signaling molecule downstream of the transforming growth factor- $\beta$ signaling pathway. It can transfer upstream signals from outside the membrane into the cell, promote the synthesis of ECM proteins, cause excessive deposition of ECM, and cause tissue fibrosis [40]. In the TGF- $\beta /$ Smad signaling pathway, the TGF- $\beta 1 / \mathrm{Smad} 3$ signaling pathway is the main and final common pathway for renal fibrosis. The activation of the SNS plays an important role in the activation of the TGF- $\beta 1 /$ Smad3 signaling pathway. Studies have shown that, on the one hand, the release of NE from the SNS can activate immune cell $\beta 2$ receptors and ultimately promote the release of TGF- $\beta 1$ [41], while on the other hand, it can also act on renal cell effectors, activate the RAAS, stimulate the activation of the TGF- $\beta$ system, and participate in the process of target organ remodeling [42]. In the present study, the expression of TGF- $\beta 1$, Smad3, and $\alpha$-SMA proteins in the RDN groups was lower than that in the sham group, suggesting that the antifibrotic effect of RDN may be related to the inhibition of the activation of the TGF- $\beta 1 / \mathrm{Smad} 3$ pathway.

In light of the above findings, we hope that further research on RDN from the adventitia of the renal artery will provide a basis for further clinical development. RDN is a potentially feasible new method not only for resistant hypertension but also for other diseases related to the overexcitement of the sympathetic nervous system.

\section{Conclusion}

RDN from the adventitia of the renal artery and RDN from the intima of the renal artery have the similar advantages of delaying high-fat-induced renal fibrosis. The antifibrotic effect of RDN may be related to inhibition of the TGF- $\beta 1 /$ Smad3 pathway.

\section{Statement of Ethics}

All experimental procedures were approved by the Animal Care Ethics Committee of Zhengzhou University (ZZULAC20180518[09]) and were conducted in accordance with the American Physiology Society's “Guides for the Care and Use of Laboratory Animals" published by the National Institutes of Health (NIH Publication No. 85-23, revised 1996).

\section{Conflict of Interest Statement}

The authors declare that they have no competing interests.

\section{Funding Sources}

This study was supported by grants from the National Natural Science Foundation of China (U1604184 to Chuanyu Gao; 81600269 to Datun Qi), the National Key Research and Development Program of China (2018YFC0114502), and the Henan Provincial and Ministry Co-construction Project (201701019). 


\section{Author Contributions}

Binbin Zhu and Yahui Liu contributed to drafting the work and revising it critically for important intellectual content, final approval of the version to be published, and agreement to be accountable for all aspects of the work in ensuring that questions related to the accuracy or integrity of any part of the work are appropriately investigated and resolved. Datun Qi, Linwei Zhao, Xiaohang Yang, Enyong Su, Zhongyu Zhu, and You Zhang contributed to acquisition and analysis of the work. Chuanyu Gao made substantial contributions to the conception or design of the work, inter- pretation of data for the work, critical revision of the work for important intellectual content, and final approval for the version to be published.

\section{Data Availability Statement}

The data that support the findings of this study are available from the corresponding author upon reasonable request. The data are not publicly available because our research team is still conducting follow-up further related research.

\section{References}

1 Yokoyama K, Tani S, Matsuo R, Matsumoto N. Association of lecithin-cholesterol acyltransferase activity and low-density lipoprotein heterogeneity with atherosclerotic cardiovascular disease risk: a longitudinal pilot study. BMC Cardiovasc Disord. 2018 Dec 5; 18(1):224.

2 Favreau F, Thuillier R, Cau J, Milin S, Manguy E, Mauco G, et al. Anti-thrombin therapy during warm ischemia and cold preservation prevents chronic kidney graft fibrosis in a DCD model. Am J Transplant. 2010 Jan; 10(1):30-9.

3 Chade AR, Mushin OP, Zhu X, RodriguezPorcel M, Grande JP, Textor SC, et al. Pathways of renal fibrosis and modulation of matrix turnover in experimental hypercholesterolemia. Hypertension. 2005 Oct;46(4):772-9.

4 Krum H, Schlaich M, Whitbourn R, Sobotka PA, Sadowski J, Bartus K, et al. Catheterbased renal sympathetic denervation for resistant hypertension: a multicentre safety and proof-of-principle cohort study. Lancet. 2009 Apr;373(9671):1275-81.

5 Lauder L, Böhm M, Mahfoud F. The current status of renal denervation for the treatment of arterial hypertension. Prog Cardiovasc Dis. 2021 Mar-Apr;65:76-83.

6 Watanabe H, Iwanaga Y, Miyaji Y, Yamamoto $\mathrm{H}$, Miyazaki S. Renal denervation mitigates cardiac remodeling and renal damage in Dahl rats: a comparison with beta-receptor blockade. Hypertens Res. 2016 Apr;39(4):217-26.

7 Bhamra-Ariza P, Rao S, Muller DW. Renal artery stenosis following renal percutaneous denervation. Catheter Cardiovasc Interv. 2014 Dec 1;84(7):1180-3.

8 Bozzani A, Arici V. Diagnosis and management of fibromuscular dysplasia. J Hypertens. 2014 Oct;32(10):2098.

9 Fischell TA, Vega F, Raju N, Johnson ET, Kent DJ, Ragland RR, et al. Ethanol-mediated perivascular renal sympathetic denervation: preclinical validation of safety and efficacy in a porcine model. EuroIntervention. 2013 May 20;9(1):140-7.

10 Castro BBA, Foresto-Neto O, Saraiva-Camara NO, Sanders-Pinheiro H. Renal lipotoxicity: insights from experimental models. Clin
Exp Pharmacol Physiol. 2021;48(12):157988.

11 Mahfoud F, Moon LB, Pipenhagen CA, Jensen JA, Pathak A, Papademetriou V, et al. Catheter-based radio-frequency renal nerve denervation lowers blood pressure in obese hypertensive swine model. J Hypertens. 2016 Sep;34(9):1854-62.

12 Gong L, Jiang F, Zhang Z, Wang X, Li H, Kuang Y, et al. Catheter-based renal denervation attenuates kidney interstitial fibrosis in a canine model of high-fat diet-induced hypertension. Kidney Blood Press Res. 2019 Jul; 44(4):628-42.

13 Zhu XY, Daghini E, Rodriguez-Porcel M, Chade AR, Napoli C, Lerman A, et al. Redoxsensitive myocardial remodeling and dysfunction in swine diet-induced experimental hypercholesterolemia. Atherosclerosis. 2007 Jul;193(1):62-9.

14 Muntner P, Coresh J, Smith JC, Eckfeldt J, Klag MJ. Plasma lipids and risk of developing renal dysfunction: the atherosclerosis risk in communities study. Kidney Int. 2000 Jul; 58(1):293-301.

15 Rockey DC, Bell PD, Hill JA. Fibrosis: a common pathway to organ injury and failure. N Engl J Med. 2015 Mar 19;372(12):1138-49.

16 Klag MJ, Whelton PK, Randall BL, Neaton JD, Brancati FL, Ford CE, et al. Blood pressure and end-stage renal disease in men. $\mathrm{N}$ Engl J Med. 1996 Jan;334(1):13-8.

17 Katayama T, Sueta D, Kataoka K, Hasegawa Y, Koibuchi N, Toyama K, et al. Long-term renal denervation normalizes disrupted blood pressure circadian rhythm and ameliorates cardiovascular injury in a rat model of metabolic syndrome. J Am Heart Assoc. 2013 Aug 23;2(4):e000197.

18 Azizi M, Schmieder RE, Mahfoud F, Weber MA, Daemen J, Davies J, et al. Endovascular ultrasound renal denervation to treat hypertension (RADIANCE-HTN SOLO): a multicentre, international, single-blind, randomised, sham-controlled trial. Lancet. 2018 Jun;391(10137):2335-45.

19 Xiao B, Liu F, Jin YH, Jin YQ, Wang L, Lu JC, et al. Renal sympathetic denervation attenuates left ventricle hypertrophy in spontane- ously hypertensive rats by suppressing the Raf/MEK/ERK signaling pathway. Clin Exp Hypertens. 2021 Feb 17;43(2):142-50.

20 Hering D, Mahfoud F, Walton AS, Krum H, Lambert GW, Lambert EA, et al. Renal denervation in moderate to severe CKD. J Am Soc Nephrol. 2012 Jul;23(7):1250-7.

21 Sanders MF, Blankestijn PJ. Chronic kidney disease as a potential indication for renal denervation. Front Physiol. 2016 Jun;7:220.

22 Ha S, Kim MJ, Kim DH, Kim BM, Chung KW, Chung HY. Short-term intake of high fat diet aggravates renal fibrosis in aged SpragueDawley rats. Exp Gerontol. 2020 Dec; 142: 111108.

23 Su X, Zhou G, Tian M, Wu S, Wang Y. Silencing of RSPO1 mitigates obesity-related renal fibrosis in mice by deactivating Wnt $/ \beta$-catenin pathway. Exp Cell Res. 2021 Aug;405(2): 112713.

$24 \mathrm{Xu} \mathrm{H}, \mathrm{Ma}$ Z, Lu S, Li R, Lyu L, Ding L, et al. Renal resistive index as a novel indicator for renal complications in high-fat diet-fed mice. Kidney Blood Press Res. 2017 Dec;42(6): 1128-40.

25 Wang H, Li J, Gai Z, Kullak-Ublick GA, Liu Z. TNF- $\alpha$ deficiency prevents renal inflammation and oxidative stress in obese mice. Kidney Blood Press Res. 2017 Jul;42(3):416-27.

26 Zhang Z, Yang K, Zeng L, Wang X, Jiang F, $\mathrm{Tu} S$, et al. Renal simplicity denervation reduces blood pressure and renal injuries in an obesity-induced hypertension dog model. Clin Exp Pharmacol Physiol. 2017 Dec; 44(12):1213-23.

27 Templin C, Jaguszewski M, Ghadri JR, Sudano I, Gaehwiler R, Hellermann JP, et al. Vascular lesions induced by renal nerve ablation as assessed by optical coherence tomography: preand post-procedural comparison with the Simplicity catheter system and the EnligHTN multi-electrode renal denervation catheter. Eur Heart J. 2013 Jul;34(28):2141-8; 2148b.

28 Sakakura K, Tunev S, Yahagi K, O’Brien AJ, Ladich E, Kolodgie FD, et al. Comparison of histopathologic analysis following renal sympathetic denervation over multiple time points. Circ Cardiovasc Interv. $2015 \mathrm{Feb} ; 8(2)$ : e001813.
Renal Interstitial Fibrosis Is Reduced in Pigs after Renal Denervation
Kidney Blood Press Res 2022;47:135-146 DOI: $10.1159 / 000521100$ 
29 Sanders MF, van Doormaal PJ, Beeftink MMA, Bots ML, Fadl Elmula FEM, Habets J, et al. Renal artery and parenchymal changes after renal denervation: assessment by magnetic resonance angiography. Eur Radiol. 2017 Sep;27(9):3934-41.

30 Atherton DS, Deep NL, Mendelsohn FO. Micro-anatomy of the renal sympathetic nervous system: a human postmortem histologic study. Clin Anat. 2012 Jul;25(5):628-33.

31 Mahfoud F, Cremers B, Janker J, Link B, Vonend $\mathrm{O}$, Ukena $\mathrm{C}$, et al. Renal hemodynamics and renal function after catheterbased renal sympathetic denervation in patients with resistant hypertension. Hypertension. 2012 Aug;60(2):419-24.

32 Zhang Z, Yang K, Zeng L, Wang X, Jiang F, $\mathrm{Tu} S$, et al. Renal simplicity denervation reduces blood pressure and renal injuries in an obesity-induced hypertension dog model. Clin Exp Pharmacol Physiol. 2017 Dec; 44(12):1213-23.
33 Bai M, Yang C, Gao C, Wang X, Liu H, Zhang $\mathrm{Y}$, et al. Effects of renal denervation from the intima and the adventitia of renal arteries on renal sympathetic nerve activity in dogs: a comparative study. Cardiology. 2015 May; 131(3):189-96

34 Kleniewska P, Piechota A, Skibska B, Gorąca A. The NADPH oxidase family and its inhibitors. Arch Immunol Ther Exp. 2012 Aug; 60(4):277-94.

35 Doerries C, Grote K, Hilfiker-Kleiner D, Luchtefeld M, Schaefer A, Holland SM, et al. Critical role of the NAD(P)H oxidase subunit p47phox for left ventricular remodeling/dysfunction and survival after myocardial infarction. Circ Res. 2007 Mar;100(6):894-903.

36 Feng Q, Lu C, Wang L, Song L, Li C, Uppada RC. Effects of renal denervation on cardiac oxidative stress and local activity of the sympathetic nervous system and renin-angiotensin system in acute myocardial infracted dogs. BMC Cardiovasc Disord. 2017 Feb;17(1):65.

37 Wang M, Han W, Zhang M, Fang W, Zhai X, Guan S, et al. Long-term renal sympathetic denervation ameliorates renal fibrosis and delays the onset of hypertension in spontaneously hypertensive rats. Am J Transl Res. 2018 Dec;10(12):4042-53.
38 Sun YB, Qu X, Caruana G, Li J. The origin of renal fibroblasts/myofibroblasts and the signals that trigger fibrosis. Differentiation. 2016 Sep;92(3):102-7.

39 Fang Q, Zheng B, Liu N, Liu J, Liu W, Huang $\mathrm{X}$, et al. Trimethylamine $\mathrm{N}$-oxide exacerbates renal inflammation and fibrosis in rats with diabetic kidney disease. Front Physiol. 2021 Jun;12:682482.

40 Meng XM, Nikolic-Paterson DJ, Lan HY. TGF- $\beta$ : the master regulator of fibrosis. Nat Rev Nephrol. 2016 Apr;12(6):325-38.

41 Elenkov IJ, Wilder RL, Chrousos GP, Vizi ES. The sympathetic nerve: an integrative interface between two supersystems: the brain and the immune system. Pharmacol Rev. 2000 Dec;52(4):595-638.

42 Rubattu S, Mennuni S, Testa M, Mennuni M, Pierelli G, Pagliaro B, et al. Pathogenesis of chronic cardiorenal syndrome: is there a role for oxidative stress? Int J Mol Sci. 2013 Nov; 14(11):23011-32. 